\title{
COHEN-MACAULAYNESS OF REES ALGEBRAS OF DIAGONAL IDEALS
}

\author{
KUEI-NUAN LIN
}

\begin{abstract}
Given two determinantal rings over a field $k$, we consider the Rees algebra of the diagonal ideal, the kernel of the multiplication map. The special fiber ring of the diagonal ideal is the homogeneous coordinate ring of the secant variety. When the Rees algebra and the symmetric algebra coincide, we show that the Rees algebra is CohenMacaulay.
\end{abstract}

1. Introduction. Determinantal rings and varieties have been a central topic of commutative algebra and algebraic geometry. We investigate blowups in the products of determinantal varieties, i.e., the Rees algebras of ideals in the determinantal rings. Rees algebras correspond to the blowups of varieties along the subvarieties in algebraic geometry. More precisely, we are interested in the Rees algebras of diagonal ideals of the determinantal rings. In this particular case, the special fiber rings of the diagonals are the homogeneous coordinate rings of the secant varieties of the determinantal rings. Therefore understanding this particular case not only lets us understand the blowup but also the secant variety.

It turns out that, for some cases, the Rees algebras and the symmetric algebras of the diagonal ideals coincide [12]. It is natural to ask if the Rees algebras are Cohen-Macaulay in those cases. In general, for a given ideal, it is very difficult to show the Cohen-Macaulayness if the ideal does not fulfill the needed hypotheses to apply the wellknown general criteria [1]. There have been various works on knowing the Cohen-Macaulayness of an ideal. Showing Cohen-Macaulayness of the Rees algebra of an ideal is even more difficult due to the general complexity of the defining ideal of the Rees algebra. There are vari-

2010 AMS Mathematics subject classification. Primary 13C40, 14M12, Secondary 13P10, 14Q15, 05E40.

Keywords and phrases. Rees algebra, secant variety, determinantal ring, symmetric algebra, Alexander dual, regularity, Cohen-Macaulay.

Received by the editors on August 23, 2013. 
ous techniques to see the Cohen-Macaulayness of Rees algebras such as using the reducing number, the analytic spread, $a$-invariant and the depth conditions of power of ideals $[\mathbf{2}, \mathbf{5}, \mathbf{1 0}, \mathbf{7}, \mathbf{8}, \mathbf{9}, \mathbf{1 1}]$. In this paper, we use the known defining equations of the Rees algebra in the work of [12] to understand the Cohen-Macaulayness of Rees algebras. The determinantal rings have nice structures; therefore, we are able to prove the Cohen-Macaulayness by looking at its initial ideal then passing it to an Alexander dual ideal. This paper continues the work of Simis and Ulrich [13], Sturmfels and Sullivant[14], and of the author [12].

We describe the ground settings of this work and outline the proof of the main theorem in Section 1. We will go through the detailed settings of this work and some basic facts of our cases in Section 2. In Section 3, we will recall some basic combinatorial properties and finish the proof of the main theorem in Section 4.

Let $k$ be a field, $2 \leq m \leq n$ integers, $X=\left[x_{i, j}\right]$ an $m \times n$ matrix of variables over $k$, and $I_{1}=I_{u_{1}}(X), I_{2}=I_{u_{2}}(X)$ the ideals of $k[X]$ generated by the $u_{1} \times u_{1}$ minors of $X$ and the $u_{2} \times u_{2}$ minors of $X$. Let $R_{1}=k[X] / I_{1}$ and $R_{2}=k[X] / I_{2}$ be two determinantal rings. We consider the diagonal ideal $\mathbf{D}$ of $S=R_{1} \otimes_{k} R_{2}$, defined via the exact sequence

$$
0 \longrightarrow \mathbf{D} \longrightarrow S \stackrel{\text { mult. }}{\longrightarrow} k[X] /\left(I_{1}+I_{2}\right) \longrightarrow 0 .
$$

The diagonal ideal $\mathbf{D}$ is generated by the images of $x_{i, j} \otimes 1-1 \otimes x_{i, j}$ in $S$. Those elements are homogeneous of degree 1 . The homogeneous coordinate ring of the embedded join variety $\mathcal{J}\left(I_{1}, I_{2}\right) \subseteq \mathbf{P}_{k}^{m n-1}$ of the determinantal varieties $V\left(I_{1}\right), V\left(I_{2}\right)$ in $\mathbf{P}_{k}^{m n-1}$ can be identified with $\mathcal{R}(\mathbf{D}) \otimes_{S} k=\mathcal{F}(\mathbf{D})$ regarding $k$ as $S / \mathfrak{m}$ where $\mathfrak{m}$ is the homogeneous maximal ideal of $S$.

The scheme Proj $(\mathcal{F}(\mathbf{D}))$ is the special fiber in the blowup Proj $(\mathcal{R}(\mathbf{D}))$ of $\operatorname{Spec}(S)$ along $V(\mathbf{D})$. In this work, we study, more broadly, the blowup rather than the special fiber.

Theorem 1.1. The Rees algebra $\mathcal{R}(\mathbf{D})$ is Cohen-Macaulay if $I_{1}$ and $\mathrm{I}_{2}$ are generated by the maximal minors of submatrices of $X$.

In [12], the defining ideals of Rees algebras of diagonal ideals have been determined in the setting of Theorem 2.1. Let $\mathcal{K}$ be the defining 
ideal of the Rees algebra of $\mathbf{D}$. By the proposition below, we deduce that $\mathcal{K}$ is Cohen-Macaulay once we show in $(\mathcal{K})$, the initial ideal of $\mathcal{K}$ via a term order, is Cohen-Macaulay.

\section{Proposition 1.2.}

(a) $[4,15.15]$ Let $R$ be a polynomial ring over a field $k,>a$ monomial order on $R, I$ an ideal of $R$ and in $(I)$ the initial ideal of $I$ with respect to the term order $>$. Let $a_{1}, \ldots, a_{r}$ be polynomials in $R$ such that in $\left(a_{1}\right), \ldots$, in $\left(a_{r}\right)$ form a regular sequence on $R /$ in $(I)$. Then $a_{1}, \ldots, a_{r}$ is a regular sequence on $R / I$.

(b) $[4,15.16,15.17]$ If $R / \operatorname{in}(I)$ is Cohen-Macaulay, then so is $R / I$.

We use combinatorial commutative algebra to show in $(\mathcal{K})$ is CohenMacaulay. With respect to a suitable term order, in $(\mathcal{K})$ is generated by square-free monomials [12]. Square-free monomial ideals in a polynomial ring are also known as Stanley-Reisner ideals. This leads us to consider Alexander dual ideals:

Theorem 1.3 ([3]). Let $J$ be a square-free monomial ideal in a polynomial ring $R$. The ring $R / J$ is Cohen-Macaulay if and only if the Alexander dual ideal $J^{*}$ has a linear free resolution.

It is sufficient to show that $(\text { in }(\mathcal{K}))^{*}$, the Alexander dual ideal of in $(\mathcal{K})$, has a linear free resolution. To do this, we find a suitable filtration starting from the Alexander dual ideal of in $(\mathcal{K})$.

2. Rees algebras of diagonal ideals. In this section, we start with the notations and write down the generators of the initial ideal of the defining ideal of diagonal ideal. Let $k$ be a field, $2 \leq m \leq n$ integers, $X_{m, n}=\left[x_{i, j}\right], Y_{m, n}=\left[y_{i, j}\right], Z_{m, n}=\left[z_{i, j}\right], m \times n$ matrices of variables over $k$. Let $s_{i}$ and $t_{i}$ be integers such that $2 \leq s_{i} \leq t_{i}, i=1,2$ and $X_{s_{1}, t_{1}}$ and $Y_{s_{2}, t_{2}}$ are the submatrices of $X$ and $Y$ coming from the first $s_{i}$ rows and first $t_{i}$ columns. $I_{1}=I_{s_{1}}\left(X_{s_{1}, t_{1}}\right)$ and $I_{2}=I_{s_{2}}\left(X_{s_{2}, t_{2}}\right)$ are the ideals of $k[X]$ generated by the maximal minors of $X_{s_{1}, t_{1}}$ and the maximal minors of $X_{s_{2}, t_{2}}$. Let $R_{1}=k[X] / I_{1}$ and $R_{2}=k[X] / I_{2}$ be two determinantal rings. We consider the diagonal ideal $\mathbf{D}$ of $R_{1} \otimes_{k} R_{2}$, 
defined via the exact sequence

$$
0 \longrightarrow \mathbf{D} \longrightarrow R_{1} \otimes_{k} R_{2} \stackrel{\text { mult. }}{\longrightarrow} k[X] /\left(I_{1}+I_{2}\right) \longrightarrow 0 .
$$

The ideal $\mathbf{D}$ is generated by the images of $x_{i, j} \otimes 1-1 \otimes x_{i, j}$ in $R_{1} \otimes_{k} R_{2}$.

We write the diagonal ideal $\mathbf{D}=\left(\left\{x_{i, j}-y_{i, j}\right\}\right)$ in

$$
S=k\left[X_{m, n}, Y_{m, n}\right] /\left(I_{s_{1}}\left(X_{s_{1}, t_{1}}\right), I_{s_{2}}\left(Y_{s_{2}, t_{2}}\right)\right) \cong R_{1} \otimes_{k} R_{2}
$$

Let $\mathcal{R}(\mathbf{D})$ be the Rees algebra of $\mathbf{D}$ over $S$ which is a subalgebra of $S[t]$. There is a natural map from $S\left[Z_{m, n}\right]$ to $\mathcal{R}(\mathbf{D}) \subseteq S[t]$ via sending $z_{i, j}$ to $\left(x_{i, j}-y_{i, j}\right) t$. Let $K$ be the kernel of this map, and write $\mathcal{R}(\mathbf{D})=S\left[Z_{m, n}\right] / K$. We consider $\phi$, a presentation matrix of $\mathbf{D}$ and the following exact sequence:

$$
S^{l} \stackrel{\phi}{\longrightarrow} S^{m n} \longrightarrow \mathbf{D} \longrightarrow 0
$$

From this, we obtain a presentation of the symmetric algebra of $\mathbf{D}$,

$$
0 \longrightarrow(\operatorname{image}(\phi))=J \longrightarrow \operatorname{Sym}\left(S^{m n}\right)=S\left[Z_{m, n}\right] \longrightarrow \operatorname{Sym}(\mathbf{D}) \longrightarrow 0 .
$$

Here $J$ is the ideal generated by the entries of the row vector $\left[z_{1,1}, z_{1,2}, \ldots, z_{1, n}, \ldots, z_{m, n}\right] \cdot \phi$. Hence,

$$
\operatorname{Sym}(\mathbf{D}) \cong S\left[Z_{m, n}\right] / J,
$$

where $J$ is generated by linear forms in the variables $z_{i, j}$. It is clear that $J \subset K$. In general, $K$ is not generated by linear forms. If $J=K$, we say $\mathbf{D}$ is an ideal of linear type. We can rewrite $\operatorname{Sym}(\mathbf{D})=S\left[Z_{m, n}\right] / J=$ $k\left[X_{m, n}, Y_{m, n}, Z_{m, n}\right] / \mathcal{J}$ and $\mathcal{R}(\mathbf{D})=k\left[X_{m, n}, Y_{m, n}, Z_{m, n}\right] / \mathcal{K}$.

Theorem 2.1. [12] Notation as above. Let $X_{a_{1} \cdots a_{s_{1}}}^{l, k}$ be the $(k-l+$ $1) \times s_{1}$ submatrix of $X$ with rows $l, l+1, \ldots, k$ and columns $a_{1}, \ldots, a_{s_{1}}$, and similarly for $Y$ and $Z$. Let

$$
\begin{aligned}
g_{i, j, l, k} & =\left|\begin{array}{cc}
z_{i, j} & z_{l, k} \\
x_{i, j}-y_{i, j} & x_{l, k}-y_{l, k}
\end{array}\right| \\
f_{a_{1}, \ldots, a_{s_{1}}} & =\sum_{q=1}^{s_{2}}(-1)^{q+1}\left|\left[\begin{array}{c}
Z^{q, q} \\
Y^{1, q-1} \\
X^{q+1, m}
\end{array}\right]_{a_{1} \cdots a_{s_{1}}}\right|,
\end{aligned}
$$

where $1 \leq a_{1}<a_{2}<\cdots<a_{s_{1}} \leq \min \left(t_{1}, t_{2}\right)$ and $1 \leq i \leq m, 1 \leq l \leq m$, $1 \leq j \leq n$ and $1 \leq k \leq n$. 
(1) Then $\mathcal{K}=\left(I_{s_{1}}\left(X_{s_{1}, t_{1}}\right), I_{s_{2}}\left(Y_{s_{2}, t_{2}}\right), g_{i, j, l, k}, f_{a_{1}, \ldots, a_{s_{1}}}\right)=\mathcal{J}$, i.e., $\mathbf{D}$ is an ideal of linear type.

(2) The initial ideal of $\mathcal{K}$ with respect to lexicographic term order and the variables ordered by $z_{i, j}>x_{l, k}>y_{p, q}$ for any $i, j, l, k, p, q$ and $x_{i, j}<x_{l, k}, y_{i, j}<y_{l, k}$ if $i>l$ or $i=l$ and $j<k$ and $z_{i, j}<z_{l, k}$ if $i>l$ or if $i=l$ and $j>k$ is generated by square-free monomials.

The generators of the Gröbner basis are collections of classes of polynomials. Each class of polynomials is complicated, and it is a huge collection. Since the main idea of this work is not about the defining equations of Rees algebra, we refer the reader to the paper [12] for detailed notation of polynomials. To simplify the notation, we write $h_{*}$ as the class of initial monomials of the class of polynomials "*." For example, we write $h_{X}$ as the class of initial monomials of $I_{s_{1}}\left(X_{s_{1}, t_{1}}\right)$ and $h_{f}$ as the class of initial monomials of $f_{a_{1}, \ldots, a_{s_{1}}}$.

Corollary 2.2. The initial ideal of

$$
\begin{gathered}
\mathcal{K}=\left(h_{X}+h_{Y}+h_{g}+h_{f}+h_{U}+h_{W}+h_{W^{p, q, l}}+h_{V}+\right. \\
\left.h_{V^{l, k, w}}+h_{H^{l, k, q}}+h_{I^{l, k, q}}+h_{k, w} I^{l, k, q}\right),
\end{gathered}
$$

where

(1) $h_{X}=\left(\left\{x_{1 a_{1}} x_{2 a_{2}} \cdots x_{s_{1} a_{s_{1}}} \mid 1 \leq a_{s_{1}}<a_{s_{1}-1}<\cdots<a_{1} \leq t_{1}\right\}\right)$,

(2) $h_{Y}=\left(\left\{y_{1 a_{1}} y_{2 a_{2}} \cdots y_{s_{2} a_{s_{2}}} \mid 1 \leq a_{s_{2}}<a_{s_{2}-1}<\cdots<a_{1} \leq t_{2}\right\}\right)$,

(3) $h_{g}=\left(\left\{z_{i j} x_{l k} \mid i<l\right.\right.$ or $i=l$ and $\left.\left.j<k,\right\}\right)$,

(4) $h_{f}=\left(\left\{z_{l q_{l}} z_{l+1 q_{l+1}} \cdots z_{k q_{k}} x_{1 a_{1}} \cdots x_{l-1 a_{l-1}} y_{1 b_{1}} \cdots y_{k-1 b_{k-1}} y_{k+1 b_{k+1}}\right.\right.$ $\cdots y_{s_{1} b_{s_{1}}} \mid 1 \leq q_{l}<q_{l+1}<\cdots<q_{k}<b_{s_{1}}<\cdots<b_{k+1}<b_{k-1}<$ $\left.\left.\cdots<b_{1}<a_{l-1}<\cdots<a_{1} \leq t_{1}, 1 \leq l<k \leq s_{2}\right\}\right)$,

(5) $h_{U}=\left(\left\{z_{p q} x_{1 a_{1}} \cdots x_{p a_{p}} y_{p+1 a_{p+1}} \cdots y_{s_{1} a_{s_{1}}} \mid p=1, \ldots, s_{1}-1,1 \leq\right.\right.$ $\left.\left.q \leq n, 1 \leq a_{s_{1}}<a_{s_{1}-1}<\cdots<a_{1} \leq t_{1}, a_{p} \leq q\right\}\right)$

(6) $h_{W}=\left(\left\{z_{p q} x_{1 a_{1}} \cdots x_{p a_{p}} y_{1 b_{1}} \cdots y_{s_{1} b_{s_{1}}} \mid p=1, \ldots, s_{1}-1,1 \leq i \leq\right.\right.$ $p, 1 \leq b_{s_{1}}<\cdots<b_{s_{2}+1}<a_{p}<\cdots<a_{1} \leq t_{1}, 1 \leq b_{s_{1}}<$ $b_{s_{1}-1}<\cdots<b_{p+2}<b_{p}<b_{p-1}<\cdots<b_{i+1}<b_{p+1}<b_{i}<$ $\cdots<b_{1} \leq t_{2}, 1 \leq b_{s_{1}}<\cdots<b_{p+2}<b_{p}<\cdots<b_{i+1}<a_{p-1}<$ $\left.\left.\cdots<a_{1} \leq t_{1}, b_{l} \neq a_{p}, l=i+1, \ldots, s_{1}, a_{p-1} \leq b_{p+1}, a_{p} \leq q\right\}\right)$,

(7) $h_{W^{p, q}, l}=\left(\left\{z_{p q} x_{1 a_{1}} \cdots x_{p a_{p}} y_{1 b_{1}} \cdots y_{p-1 b_{p-1}} y_{p b_{p}} y_{p+1 b_{p+1}} y_{p+1 b_{p+1}^{\prime}}\right.\right.$ $\cdots y_{v b_{v}} y_{v b_{v}^{\prime}} y_{v+1 b_{v+1}} \cdots y_{s_{1} b_{s_{1}}} \mid 1 \leq p \leq m, 1 \leq q \leq n, v=$ 
$p+1, \ldots, s_{2}-1,1 \leq b_{s_{1}}<\cdots<b_{s_{2}+1}<a_{p}<\cdots<a_{1} \leq$ $t_{1}, a_{p} \leq q, b_{s_{2}+1}<b_{s_{2}}<\cdots<b_{v+2}<b_{v}^{\prime}<\cdots<b_{p+1}^{\prime}<$ $b_{p-1}<\cdots<b_{i+1}<a_{p-1} \leq b_{p+1}<b_{i}<\cdots<b_{1} \leq t_{2}, 1 \leq i \leq$ $p, b_{l}^{\prime} \neq a_{p}, l \geq i+1, b_{v-1}^{\prime} \leq b_{v+1}, b_{s_{1}}<\cdots<b_{s_{2}+1}<b_{s_{2}}<$ $\cdots<b_{v+2}<b_{v+1}<b_{v}<b_{v-1}<\cdots<b_{p+2}<b_{p+1}<a_{p}<$ $\left.\left.a_{p-1} \leq b_{p}, b_{r}^{\prime} \leq b_{r+2}<b_{r+1}, r=p, \ldots, v-2\right\}\right)$,

(8) $h_{V}=\left(\left\{z_{l q_{l}} z_{l+1 q_{l+1}} \cdots z_{k q_{k}} x_{1 a_{1}} \cdots x_{l-1 a_{l-1}} y_{1 b_{1}} \cdots y_{k-1 b_{k-1}} y_{k b_{k}}\right.\right.$ $y_{k+1 b_{k+1}} \cdots y_{s_{1} b_{s_{1}}} \mid 1 \leq l<k \leq s_{2}, 1 \leq q_{l}<q_{l+1}<\cdots<$ $q_{k}<b_{s_{1}}<\cdots<b_{k+2}<b_{k}<b_{k-1}<\cdots<b_{1}<a_{l-1}<\cdots<$ $\left.a_{1} \leq t_{1}, \quad b_{k-1} \leq b_{k+1} \leq t_{2}-k+1\right\}$

(9) $h_{V^{l, k, w}}=\left(\left\{z_{l q_{l}} \cdots z_{k q_{k}} x_{1 a_{1}} \cdots x_{l-1 a_{l-1}} y_{1 b_{1}} \cdots y_{k+1 b_{k+1}} y_{k+1 b_{k+1}^{\prime}}\right.\right.$ $\cdots y_{w b_{w}} y_{w b_{w}^{\prime}} y_{w+1 b_{w+1}} \cdots y_{s_{1} b_{s_{1}}} \mid 1 \leq l \leq k \leq s_{2}, 1 \leq p_{l}<$ $\cdots<p_{k}<b_{s_{1}}<\cdots<b_{s_{2}+1}<\cdots<b_{k+1}<b_{k-1}<b_{k}<$ $b_{k-2}<\cdots<b_{1}<a_{l-1}<\cdots<a_{1} \leq t_{1}, w=k, \ldots, s_{2}-1,1 \leq$ $b_{s_{2}}<\cdots<b_{w+2}<b_{w}^{\prime}<b_{w-1}^{\prime}<\cdots<b_{k}^{\prime}<b_{k-1}<b_{k-2} \cdots<$ $\left.\left.b_{1} \leq t_{2}, b_{w-1}^{\prime} \leq b_{w+1}, b_{r}^{\prime} \leq b_{r+2}<b_{r+1}, r=k, \ldots, l-2\right\}\right)$,

(10) $h_{H^{l, k, q}}=\left(\left\{z_{l-1, q} z_{l, q_{l}} \cdots z_{k q_{k}} x_{1 a_{1}} \cdots x_{l-2, a_{l-1}} y_{1 b_{1}} \cdots y_{k-1 b_{k-1}}\right.\right.$ $y_{k+1 b_{k+1}} \cdots y_{s_{1} b_{s_{1}}} \mid 1 \leq l \leq k \leq s_{2}, 1 \leq q \leq n, 1 \leq q_{l}<$ $\cdots<q_{k}<b_{s_{1}}<\cdots<b_{k+1}<b_{k-1}<\cdots<b_{1}<a_{l-2}<\cdots<$ $\left.\left.a_{1} \leq t_{1}, q_{l}<a_{l-1} \leq q<b_{1}\right\}\right)$,

(11) $h_{I^{l, k, q}}=\left(\left\{z_{l-1, q} z_{l, q_{l}} \cdots z_{k q_{k}} x_{1 a_{1}} \cdots x_{l-2, a_{l-1}} y_{1 b_{1}} \cdots y_{k-1 b_{k-1}} y_{k b_{k}}\right.\right.$ $y_{k+1 b_{k+1}} \cdots y_{s_{1} b_{s_{1}}} \mid 1 \leq l \leq k \leq s_{2}, 1 \leq q \leq n, 1 \leq q_{l}<\cdots<$ $q_{k}<b_{s_{1}}<\cdots<b_{k+1}<b_{k-1}<b_{k}<b_{k-2}<\cdots<b_{1}<a_{l-2}<$ $\left.\left.\cdots<a_{1} \leq t_{1}, q_{l}<a_{l-1} \leq q<b_{1}\right\}\right)$

(12) $h_{k, w} I^{l, k, q}=\left(\left\{z_{l-1, q} z_{l, q_{l}} \cdots z_{k q_{k}} x_{1 a_{1}} \cdots x_{l-2, a_{l-1}} y_{1 b_{1}} \cdots y_{k b_{k}} y_{k b_{k}^{\prime}}\right.\right.$ $\cdots y_{w b_{w}} y_{w b_{w}^{\prime}} y_{w+1 b_{w+1}} \cdots y_{s_{1} b_{s_{1}}} \mid 1 \leq l \leq k \leq s_{2}, 1 \leq q \leq$ $n, 1 \leq q_{l}<\cdots<q_{k}<b_{s_{1}}<\cdots<b_{k+1}<b_{k-1}<b_{k}<b_{k-2}<$ $\cdots<b_{1}<a_{l-2}<\cdots<a_{1} \leq t_{1}, q_{l}<a_{l-1} \leq q<b_{1}, w=$ $k, \cdots, s_{2}-1,1 \leq b_{s_{2}}<\cdots<b_{w+2}<b_{w}^{\prime}<b_{w-1}^{\prime}<\cdots<b_{k}^{\prime}<$ $b_{k-1}<b_{k-2} \cdots<b_{1} \leq t_{2}, b_{w-1}^{\prime} \leq b_{w+1}, b_{r}^{\prime} \leq b_{r+2}<b_{r+1}, r=$ $k, \cdots, l-2\})$.

3. Alexander dual. We turn our focus on finding the Alexander dual of given monomial ideals in this section. Also we show techniques needed for the proof of the main lemma including how to use a filtration of a square-free monomial ideal to show it has linear free resolution. Notice, from Corollary 2.2, the initial ideal of $\mathcal{K}$ is generated by square- 
free monomials since each class of monomials is a product of variables and those variables either coming from different rows or at the same row with different column indices. We know that an ideal generated by square-free monomials defines a Stanley-Reisner ring. Hence, we can find the Alexander dual ideal of this ideal [1]. We recall the definition of the Alexander dual ideal.

Definition 3.1. If $I$ is an ideal of $R=k\left[x_{1}, \ldots, x_{n}\right]$ generated by square-free monomials $\left(f_{1}, \ldots, f_{l}\right)$, then the Alexander dual ideal $I^{*}$ of $I$ is $\cap_{i} P_{f_{i}}$, where for any square-free monomial $f=x_{i_{1}} \cdots x_{i_{r}}$, $P_{f}=\left(x_{i_{1}}, \ldots, x_{i_{r}}\right)$.

From Corollary 2.2, the initial ideal of $\mathcal{K}$ is generated by classes of monomials having very similar forms as the ones in the following lemma; hence, we find Alexander dual ideals of those kind first. Then the Alexander dual ideal of in $(\mathcal{K})$ is generated by the intersection of similar forms by the definition of Alexander dual ideals.

Lemma 3.2. Let $R=k[X]$, where $X=\left[x_{i, j}\right], i=1, \ldots, m, j=$ $1, \ldots, n$ and $1 \leq a_{0} \leq l<m \leq n$. Let $I$ be the ideal generated by $\left\{x_{1, a_{1}} x_{2, a_{2}} x_{3, a_{3}} \cdots x_{m, a_{m}}\right\}$ with $1 \leq a_{0} \leq a_{1}<a_{2}<\cdots<a_{l} \leq a_{l+1}<$ $\cdots<a_{m} \leq n$. Then $I^{*}$, the Alexander dual ideal of $I$, is generated by

$$
\begin{aligned}
&\left\{\prod_{i_{1}=a_{0}}^{k_{1}} x_{1, i_{1}} \prod_{i_{2}=k_{1}+2}^{k_{2}} x_{2, i_{2}} \ldots\right. \\
& \prod_{i_{l}=k_{l-1}+2}^{k_{l}} x_{l, i_{l}} \prod_{i_{l+1}=k_{l}+1}^{k_{l+1}} x_{l+1, i_{l+1}} \prod_{i_{l+2}=k_{l+1}+2}^{k_{l+2}} x_{l+2, i_{l+2}} \ldots \\
&\left.\prod_{i_{m}=k_{m-1}+2}^{n} x_{m, i_{m}}\right\},
\end{aligned}
$$

where $a_{0}-1 \leq k_{1}<k_{2}<k_{3} \cdots<k_{l} \leq k_{l+1}<\cdots<k_{m-1}<n$.

Proof. Without lost of generality, we may assume $l=1$. Inducting on $m$, we consider $m=2$ and $m=3$ first. When $m=2, I=$ 


$$
\begin{aligned}
\left(\left\{x_{1, a_{1}} x_{2, a_{2}} \mid a_{0} \leq a_{1} \leq a_{2} \leq n\right\}\right) . \text { Now } \\
I^{*}=\bigcap_{a_{0} \leq a_{1} \leq a_{2} \leq n}\left(x_{1, a_{1}}, x_{2, a_{2}}\right) \\
=\bigcap_{a_{0} \leq a_{1} \leq n}\left(\bigcap_{a_{1} \leq a_{2} \leq n}\left(x_{1, a_{1}}, x_{2, a_{2}}\right)\right) \\
=\bigcap_{a_{0} \leq a_{1} \leq n}\left(x_{1, a_{1}}, \prod_{i_{2}=a_{1}}^{n} x_{2, i_{2}}\right) \\
=\left(\left\{\prod_{i_{1}=a_{0}}^{k_{1}} x_{1, i_{1}} \prod_{i_{2}=k_{1}+1}^{n} x_{2, i_{2}} \mid a_{0}-1 \leq k_{1} \leq n\right\}\right) .
\end{aligned}
$$

When $m=3$, we have $I=\left(\left\{x_{1, a_{1}} x_{2, a_{2}} x_{3, a_{3}} \mid a_{0} \leq a_{1} \leq a_{2}<a_{3} \leq n\right\}\right)$. Now

$$
\begin{aligned}
I^{*} & =\bigcap_{a_{0} \leq a_{1}<n}\left(\bigcap_{a_{1} \leq a_{2}<n}\left(\bigcap_{a_{2}<a_{3} \leq n}\left(x_{1, a_{1}}, x_{2, a_{2}}, x_{3, a_{3}}\right)\right)\right) \\
& =\bigcap_{a_{0} \leq a_{1}<n}\left(\bigcap_{a_{1} \leq a_{2}<n}\left(x_{1, a_{1}}, x_{2, a_{2}}, \prod_{i_{3}=a_{2}+1}^{n} x_{3, i_{3}}\right)\right) \\
& =\bigcap_{a_{0} \leq a_{1}<n}\left(x_{1, a_{1}},\left\{\prod_{i_{2}=a_{1}}^{k_{2}} x_{2, i_{2}} \prod_{i_{3}=k_{2}+2}^{n} x_{3, i_{3}} \mid a_{1}-1 \leq k_{2}<n\right\}\right) \\
& =\left(\left\{\prod_{i_{1}=a_{0}}^{k_{1}} x_{1, i_{1}} \prod_{i_{2}=k_{1}+1}^{k_{2}} x_{2, i_{2}} \prod_{i_{3}=k_{2}+2}^{n} x_{3, i_{3}} \mid a_{0}-1 \leq k_{1} \leq k_{2}<n\right\}\right) .
\end{aligned}
$$

When $m>3$, we have

$$
\begin{aligned}
I^{*}= & \left.\bigcap_{a_{0} \leq a_{1} \leq a_{2}<a_{3}<\cdots<a_{m} \leq n}\left(\bigcap_{1, a_{1}}, x_{2, a_{2}}, \ldots, x_{m, a_{m}}\right)\right) \\
& \left(\bigcap_{a_{1} \leq a_{2}<a_{3}<\cdots<a_{m} \leq n} \bigcap_{a_{0} \leq a_{1} \leq a_{2}<a_{3}<\cdots<a_{m} \leq n}^{n} x_{m, i_{m}} \mid\right. \\
& \left(x_{1, a_{1}},\left\{\prod_{i_{2}=a_{1}}^{k_{2}} x_{2, i_{2}} \prod_{i_{3}=k_{2}+2}^{k_{3}} x_{3, i_{3}} \cdots \prod_{i_{m}=k_{m-1}+2} x\right.\right.
\end{aligned}
$$




$$
\begin{aligned}
& \left.\left.a_{1}-1 \leq k_{2}<k_{3}<\cdots<k_{m-1}<n\right\}\right) \\
= & \left(\left\{\prod_{i_{1}=a_{0}}^{k_{1}} x_{1, i_{1}} \prod_{i_{2}=k_{1}+1}^{k_{2}} x_{2, i_{2}} \prod_{i_{3}=k_{2}+2}^{k_{3}} x_{3, i_{3}} \cdots \prod_{i_{m}=k_{m-1}+2}^{n} x_{m, i_{m}} \mid\right.\right. \\
& \left.\left.a_{0}-1 \leq k_{1} \leq k_{2}<k_{3}<\cdots<k_{m-1}<n\right\}\right)
\end{aligned}
$$

where the second equality comes from the induction.

Having the Alexander dual ideal of in $(\mathcal{K})$, we can use Theorem 1.3 to show that in $(\mathcal{K})$ is Cohen-Macaulay once we show that the Alexander dual ideal has a linear free resolution. We recall the definition of a linear free resolution and the regularity of an ideal.

\section{Definition 3.3.}

(a) Let

$$
\mathbf{F}: \cdots \longrightarrow F_{i} \longrightarrow F_{i-1} \longrightarrow \cdots \longrightarrow F_{0}
$$

be a minimal homogeneous free resolution of an ideal $I$ in a $\operatorname{ring} R=k\left[x_{1}, \ldots, x_{n}\right]$ with $F_{i}=\oplus_{j} R\left(-a_{i j}\right)$. We say $I$ has a linear free resolution if $a_{i j}=a_{i}$ and $a_{i+1}=a_{i}+1$.

(b) The regularity of $I$ is defined as $\operatorname{reg}(I)=\max _{i, j}\left\{a_{i j}-i\right\}$.

Fact 3.4. If all the minimal homogeneous generators of $I$ have the same degree, $d$, then $I$ has a linear free resolution if and only if $\operatorname{reg}(I)=d$.

We will show that $(\text { in }(\mathcal{K}))^{*}$ is generated in the same degree as $d$ and $\operatorname{reg}(\operatorname{in}(\mathcal{K}))^{*}=d$. The structure of $(\operatorname{in}(\mathcal{K}))^{*}$ is very similar to the one in the following proposition, and we show its Alexander dual has a linear free resolution. Later, we will use the same technique again and again to show $(\text { in }(\mathcal{K}))^{*}$ is generated in the same degree $d$ and $\operatorname{reg}(\text { in }(\mathcal{K}))^{*}=d$. In this way, we can reduce the confusion of complex notations from $(\text { in }(\mathcal{K}))^{*}$.

Proposition 3.5. Let $R=k[X]$, where $X=\left[x_{i, j}\right], i=1, \ldots, m$, $j=1, \ldots, n$. Let $I$ be the ideal generated by $\left\{x_{1, a_{1}} x_{2, a_{2}} x_{3, a_{3}} \cdots x_{m, a_{m}}\right\}$ with $1 \leq a_{1}<a_{2}<\cdots<a_{l} \leq a_{l+1}<\cdots<a_{m}<n$ for some 
$1 \leq l \leq m-1$. Then $I^{*}$, the Alexander dual ideal of I has a linear free resolution.

Proof. From Lemma 3.2, we see that $I^{*}$ is generated by elements of degree $n-(m-2)$, denoted by $\operatorname{deg}\left(I^{*}\right)$. Using Fact 3.4, it's sufficient to show that $\operatorname{reg}\left(I^{*}\right)=\operatorname{deg}\left(I^{*}\right)=n-(m-2)$. We will induct on $n$ to show that there is a filtration on $I^{*}$ such that the quotient is linear up to degree shifting.

We write down $I^{*}$ first,

$$
\begin{array}{r}
I^{*}=\left(\left\{\prod_{i_{1}=1}^{k_{1}} x_{1, i_{1}} \prod_{i_{2}=k_{1}+2}^{k_{2}} x_{2, i_{2}} \ldots \prod_{i_{l}=k_{l-1}+2}^{k_{l}} x_{i, i_{l}} \prod_{i_{l+1}=k_{l}+1}^{k_{l+1}} x_{i, i_{l+1}}\right.\right. \\
\left.\left.\ldots \prod_{i_{m}=k_{m-1}+2}^{n} x_{m, i_{m}}\right\}\right)
\end{array}
$$

with $0 \leq k_{1}<k_{2}<\cdots<k_{l} \leq k_{l+1}<\cdots<k_{m-1}<n$.

When $m=n$, we have $0 \leq k_{1}<k_{2}<\cdots<k_{l} \leq k_{l+1}<\cdots<k_{m-1}<$ $m$. Without lost of generality, we assume $l=1$. Hence,

$$
\begin{aligned}
I^{*}=\left(x_{1,1} x_{1,2},\left\{x_{1,1} x_{i, i} \mid\right.\right. & i=2, \ldots, m\} \\
& \left.\left\{x_{i, i-1} x_{j, j} \mid i=2, \ldots, m, j=i, \ldots, m\right\}\right) .
\end{aligned}
$$

Now look at $x_{m, m}$; the terms $x_{i, i-1} x_{m, m}$ for $i=2, \ldots, m-1$ and $x_{1,1} x_{m, m}$ are multiples of $x_{m, m}$. Also, $x_{i, i-1} x_{j, j}$ for $i=2, \ldots, m-1$, $j=i, \ldots, m-1$ is divisible by $x_{i, i-1}$ and $x_{1,1} x_{1,2}$ and $x_{1,1} x_{i, i}$ for $i=2, \ldots, m-1$ is divisible by $x_{1,1}$. We can rewrite

$$
\begin{aligned}
I^{*}=\left(\left\{x_{i, i-1}\left(x_{i, i}, \ldots, x_{m, m}\right) \mid i=2, \ldots, m-1\right\},\right. & \\
& \left.x_{1,1}\left(x_{1,2}, x_{2,2}, \ldots, x_{m, m}\right)\right) .
\end{aligned}
$$

Let $J_{l}=\left(\left\{x_{i, i-1}\left(x_{i, i}, \ldots, x_{m, m}\right) \mid i=l, \ldots, m-1\right\}, x_{1,1}, x_{2,1}, x_{3,2}, \ldots\right.$, $\left.x_{l-1, l-2}\right)$ where $l=1, \ldots, m$ and $I^{*}=J_{1}$. One can see that $J_{m}=$ $\left(\left\{x_{i, i-1} \mid i=2, \ldots, m-1\right\}, x_{1,1}\right)$ is generated by a regular sequence of degree 1 ; hence, it has reg $J_{m}=1$. Furthermore, $J_{l} \supseteq J_{l+1}$ for $l=2, \ldots, m-1$. 
We claim that those $J_{i}$ 's are the filtrations we need and show $\operatorname{reg}\left(J_{l+1} / J_{l}\right)=1$ for $l=1, \ldots, m-1$. Therefore, $\operatorname{reg}\left(J_{l}\right)=2=$ $m-(m-2)$ for $l=1, \ldots, m-1$. Notice $J_{l}$ can be rewritten as

$$
\begin{aligned}
J_{l}= & \left(\left\{x_{i, i-1}\left(x_{i, i}, \ldots, x_{m, m}\right)|| i=l, \ldots, m-1\right\}\right. \\
& \left.x_{1,1}, x_{2,1}, x_{3,2}, \ldots, x_{l-1, l-2}\right) \\
= & \left(\left\{x_{i, i-1}\left(x_{i, i}, \ldots, x_{m, m}\right) \mid i=l+1, \ldots, m-1\right\},\right. \\
& x_{l, l-1}\left(x_{l, l}, \ldots, x_{m, m}\right), \\
& \left.\quad x_{1,1}, x_{2,1}, x_{3,2}, \ldots, x_{l-1, l-2}\right) .
\end{aligned}
$$

Then

$$
\begin{gathered}
J_{l+1} / J_{l}=\left(x_{l, l-1}\right) /\left(x_{l, l-1} \cap\left(\left\{x_{i, i-1}\left(x_{i, i}, \ldots, x_{m, m}\right) \mid i=l+1, \ldots, m-1\right\}\right),\right. \\
\left.x_{l, l-1} \cap\left(x_{1,1}, x_{2,1}, x_{3,2}, \ldots, x_{l-1, l-2}\right), x_{l, l-1}\left(x_{l, l}, \ldots, x_{m, m}\right)\right) \\
=\left(x_{l, l-1}\right) / x_{l, l-1}\left(x_{l, l}, \ldots, x_{m, m}, x_{1,1}, x_{2,1}, x_{3,2}, \ldots, x_{l-1, l-2}\right) .
\end{gathered}
$$

Since $x_{l, l-1}$ is regular over

$$
R /\left(x_{l, l}, \ldots, x_{m, m}, x_{1,1}, x_{2,1}, x_{3,2}, \ldots, x_{l-1, l-2}\right),
$$

we obtain

$$
\begin{aligned}
& \operatorname{reg}\left(\left(x_{l, l-1}\right) / x_{l, l-1}\left(x_{l, l}, \ldots, x_{m, m}, x_{1,1}, x_{2,1}, x_{3,2}, \ldots, x_{l-1, l-2}\right)\right) \\
& \quad=\operatorname{reg}\left(R /\left(x_{l, l}, \ldots, x_{m, m}, x_{1,1}, x_{2,1}, x_{3,2}, \ldots, x_{l-1, l-2}\right)\right)+1=1,
\end{aligned}
$$

and therefore $\operatorname{reg}\left(J_{l+1} / J_{l}\right)=1$ for all $l=1, \ldots, m-1$. We are now done with the case $m=n$.

For the induction part, we write $I^{*}:=I_{n}^{*}$ when $X$ is an $m \times n$ matrix. We assume $\operatorname{reg}\left(I_{n-1}^{*}\right)=n-1-(m-2)=\operatorname{deg}\left(I_{n-1}^{*}\right)$ and $m<n$. Before we write down the filtration, we state some facts of relations of generators of $I_{n}^{*}$,

$$
\begin{array}{r}
\left\{\prod_{i_{1}=1}^{k_{1}} x_{1, i_{1}} \prod_{i_{2}=k_{1}+2}^{k_{2}} x_{2, i_{2}} \ldots \prod_{i_{l}=k_{l-1}+2}^{k_{l}} x_{i, i_{l}} \prod_{i_{l+1}=k_{l}+1}^{k_{l+1}} x_{i, i_{l+1}}\right. \\
\left.\ldots \prod_{i_{m}=k_{m-1}+2}^{n} x_{m, i_{m}}\right\} .
\end{array}
$$


Those relations will help us show quotients of the filtration are what we need. Let's consider the variable $x_{m, n}$. When $k_{m-1}=n-2$,

$$
\left\{\prod_{i_{1}=1}^{k_{1}} x_{1, i_{1}} \prod_{i_{2}=k_{1}+1}^{k_{2}} x_{2, i_{2}} \prod_{i_{3}=k_{2}+2}^{k_{3}} x_{3, i_{3}} \ldots \prod_{i_{m-1}=k_{m-2}+2}^{k_{m-1}=n-2} x_{m-1, i_{m-1}} x_{m, n}\right\}
$$

is divisible by $x_{m, n}$, and we write

$$
\begin{aligned}
& A=\left\{\left(\prod_{i_{1}=1}^{k_{1}} x_{1, i_{1}} \prod_{i_{2}=k_{1}+1}^{k_{2}} x_{2, i_{2}} \prod_{i_{3}=k_{2}+2}^{k_{3}} x_{3, i_{3}}\right.\right. \\
& \left.\left.\cdots \prod_{i_{m-1}=k_{m-2}+2}^{n-2} x_{m-1, i_{m-1}}\right) x_{m, n}\right\}=A^{\prime} x_{m, n},
\end{aligned}
$$

where $0 \leq k_{1} \leq k_{2}<k_{3}<\cdots<k_{m-1}=n-2$. When $k_{m-1}=n-1$,

$$
\prod_{i_{1}=1}^{k_{1}} x_{1, i_{1}} \prod_{i_{2}=k_{1}+1}^{k_{2}} x_{2, i_{2}} \prod_{i_{3}=k_{2}+2}^{k_{3}} x_{3, i_{3}} \ldots \prod_{i_{m}=k_{m-1}+2}^{n} x_{m, i_{m}}
$$

is not divisible by $x_{m, n}$, we write

$$
B=\left\{\prod_{i_{1}=1}^{k_{1}} x_{1, i_{1}} \prod_{i_{2}=k_{1}+1}^{k_{2}} x_{2, i_{2}} \prod_{i_{3}=k_{2}+2}^{k_{3}} x_{3, i_{3}} \ldots \prod_{i_{m}=k_{m-2}+2}^{n-1} x_{m-1, i_{m-1}}\right\},
$$

where $0 \leq k_{1} \leq k_{2}<k_{3}<\ldots<k_{m-2}<k_{m-1}=n-1$. One can see

$$
B=A^{\prime}\left(\left\{x_{j, j-1+(n-1-(m-2))} \mid 1<j<m\right\}, x_{1, n-1-(m-2)}\right) .
$$

When $k_{m-1} \leq n-3$, we write

$$
\begin{aligned}
C & \left.=\left\{\prod_{i_{1}=1}^{k_{1}} x_{1, i_{1}} \prod_{i_{2}=k_{1}+1}^{k_{2}} x_{2, i_{2}} \prod_{i_{3}=k_{2}+2}^{k_{3}} x_{3, i_{3}} \ldots \prod_{i_{m}=k_{m-1}+2}^{n-1} x_{m, i_{m}}\right) x_{m, n}\right\} \\
& =C^{\prime} x_{m, n}
\end{aligned}
$$

where $0 \leq k_{1} \leq k_{2}<k_{3}<\cdots<k_{m-2}<k_{m-1} \leq n-3$. Hence, $I_{n}^{*}=(A, B, C)$. Let

$$
C^{\prime \prime}=\left\{\prod_{i_{1}=1}^{k_{1}} x_{1, i_{1}} \prod_{i_{2}=k_{1}+1}^{k_{2}} x_{2, i_{2}} \prod_{i_{3}=k_{2}+2}^{k_{3}} x_{3, i_{3}} \ldots \prod_{i_{m}=k_{m-1}+2}^{n-1} x_{m, i_{m}}\right\},
$$


where $0 \leq k_{1} \leq k_{2}<k_{3}<\cdots<k_{m-2}<k_{m-1}<n-3$; then $C$ can be written as

$$
C=C^{\prime \prime} x_{m, n}\left(\left\{x_{j, j-1+(n-1-(m-2))-1} \mid 1<j<m\right\}, x_{1, n-1-(m-2)}\right) .
$$

Notice $I_{n-1}^{*}=\left(A^{\prime}, C^{\prime}\right)$ and the elements of $A^{\prime}$ and $C^{\prime}$ have the same degree. Let

$$
A^{\prime \prime}=\left\{\prod_{i_{1}=1}^{k_{1}} x_{1, i_{1}} \prod_{i_{2}=k_{1}+1}^{k_{2}} x_{2, i_{2}} \prod_{i_{3}=k_{2}+2}^{k_{3}} x_{3, i_{3}} \ldots \prod_{i_{m}=k_{m-2}+2}^{n-2} x_{m-1, i_{m-1}}\right\},
$$

where $0 \leq k_{1} \leq k_{2}<k_{3}<\cdots<k_{m-2}<k_{m-1}=n-3$. Then we write

$$
A^{\prime}=A^{\prime \prime}\left(\left\{x_{j, j-1+n-m-1} \mid 1<j<m\right\}, x_{1, n-2-(m-2)}\right) .
$$

Now, looking at $C^{\prime}$, we obtain

$$
\begin{aligned}
C^{\prime}=\left(\left\{\left(\prod_{i_{1}=1}^{k_{1}} x_{1, i_{1}}\right.\right.\right. & \prod_{i_{2}=k_{1}+1}^{k_{2}} x_{2, i_{2}} \prod_{i_{3}=k_{2}+2}^{k_{3}} x_{3, i_{3}} \\
& \left.\left.\left.\ldots \prod_{i_{m}=k_{m-1}+2}^{n-2} x_{m, i_{m}}\right) x_{m, n-1}\right\}, A^{\prime \prime} x_{m, n-1}\right),
\end{aligned}
$$

where $0 \leq k_{1} \leq k_{2}<k_{3}<\cdots<k_{m-2}<k_{m-1}<n-3$. Hence,

$$
\begin{aligned}
\left(A^{\prime}\right) \cap\left(C^{\prime}\right)= & \left(A^{\prime \prime}\left(\left\{x_{j, j-1+n-m-1} \mid 1<j<m\right\}, x_{1, n-2-(m-2)}\right)\right) \cap \\
& \left\{\left(\prod_{i_{1}=1}^{k_{1}} x_{1, i_{1}} \prod_{i_{2}=k_{1}+1}^{k_{2}} x_{2, i_{2}} \prod_{i_{3}=k_{2}+2}^{k_{3}} x_{3, i_{3}}\right.\right. \\
& \left.\left.\quad \ldots \prod_{i_{m}=k_{m-1}+2}^{n-2} x_{m, i_{m}}\right) x_{m, n-1}\right\}, \\
& A^{\prime \prime}\left(\left\{x_{j, j-1+n-m-1} \mid 1<j<m\right\}, x_{1, n-2-(m-2)}\right) \\
\cap & \left(A^{\prime \prime} x_{m, n-1}\right) \\
= & \left(A^{\prime} x_{m, n-1}\right) \\
= & \left(C^{\prime}\left(\left\{x_{j, j-1+n-m-1} \mid 1<j<m\right\}, x_{1, n-2-(m-2)}\right)\right) .
\end{aligned}
$$


On the other hand, we have

$$
\begin{aligned}
\left(A^{\prime}\right) \cap\left(C^{\prime} x_{m, n}\right)= & \left(A^{\prime}\right) \cap\left(C ^ { \prime \prime } x _ { m , n } \left(\left\{x_{j, j-1+n-m}||\right.\right.\right. \\
& \left.\left.1<j<m\}, x_{1, n-1-(m-2)}\right)\right) \\
\subset & \left(A^{\prime}\left(\left\{x_{j, j-1+n-m} \mid 1<j<m\right\}, x_{1, n-m+1}\right)\right) .
\end{aligned}
$$

Consider the following filtration:

$$
\begin{aligned}
I_{n}^{*}= & (C, B, A)=\left(C^{\prime} x_{m, n}, A^{\prime}\left(\left\{x_{j, j-1+n-m}\right.\right.\right. \\
& \left.\left.\mid 1<j<m\}, x_{1, n-m+1}\right), A^{\prime} x_{m, n}\right) \\
\subset & \left(C^{\prime} x_{m, n}, A^{\prime}\right) \\
\subset & \left(C^{\prime}, A^{\prime}\right)=I_{n-1}^{*} .
\end{aligned}
$$

By using the intersections we obtain above, the relations of regularity follow:

$$
\begin{aligned}
& \operatorname{reg}\left(\left(C^{\prime}, A^{\prime}\right) /\left(C^{\prime} x_{m, n}, A^{\prime}\right)\right) \\
&= \operatorname{reg}\left(\left(C^{\prime}\right) /\left(\left(C^{\prime}\right) \cap\left(A^{\prime}\right), C^{\prime} x_{m, n}\right)\right) \\
&= \operatorname{reg}\left(\left(C^{\prime}\right) /\left(C ^ { \prime } \left(\left\{x_{j, j-1+n-m-1}\right.\right.\right.\right. \\
&\left.\left.\quad \mid 1<j<m\}, x_{1, n-2-(m-2)}, x_{m, n}\right)\right) \\
&= \operatorname{reg}\left(R /\left(\left\{x_{j, j-1+n-m-1} \mid 1<j<m\right\}, x_{1, n-2-(m-2)}, x_{m, n}\right)\right. \\
&+\operatorname{deg}\left(C^{\prime}\right) \\
&= \operatorname{deg}\left(C^{\prime}\right) \\
&= n-(m-2)-1 .
\end{aligned}
$$

Since reg $\left(C^{\prime}, A^{\prime}\right)=\operatorname{reg}\left(I_{n-1}^{*}\right)=n-1-(m-2)$, by the induction hypothesis, we use the short exact sequence of regularity to obtain $\operatorname{reg}\left(C^{\prime} x_{m, n}, A^{\prime}\right)=n-(m-2)$. Similarly,

$$
\begin{aligned}
\operatorname{reg}\left(\left(C^{\prime} x_{m, n}, A^{\prime}\right) /\left(C^{\prime} x_{m, n}, A^{\prime}\left(\left\{x_{j, j-1+n-m} \mid\right.\right.\right.\right. & \\
& \left.\left.\left.1<j<m\}, x_{1, n-m+1}\right), A^{\prime} x_{m, n}\right)\right) \\
= & \operatorname{reg}\left(\left(A^{\prime}\right) /\left(\left(A^{\prime}\right) \cap\left(C^{\prime} x_{m, n}\right), A^{\prime}\left(\left\{x_{j, j-1+n-m} \mid\right.\right.\right.\right. \\
& \left.\left.\left.1<j<m\}, x_{1, n-m+1}\right), A^{\prime} x_{m, n}\right)\right) \\
= & \operatorname{reg}\left(\left(A^{\prime}\right) /\left(A ^ { \prime } \left(\left\{x_{j, j-1+n-m} \mid 1<j<m\right\},\right.\right.\right. \\
& \left.\left.\quad x_{1, n-m+1}, x_{m, n}\right)\right) \\
= & \operatorname{reg}\left(R /\left(A ^ { \prime } \left(\left\{x_{j, j-1+n-m} \mid 1<j<m\right\}, x_{1, n m+1},\right.\right.\right.
\end{aligned}
$$




$$
\begin{gathered}
\left.\left.\left.x_{m, n}\right)\right)\right)+\operatorname{deg}\left(A^{\prime}\right) \\
=\operatorname{deg}\left(A^{\prime}\right)=n-(m-2)-1 .
\end{gathered}
$$

Hence reg $\left(I_{n}^{*}\right)=n-(m-2)$.

4. Proof of the main theorem. In this section, we find the Alexander dual ideal of in $(\mathcal{K})$, and we find the regularity of the Alexander dual in Lemma 4.2. Then we finish the proof of the Theorem 1.1 and end this section by Example 4.3. In order to simplify the notations of the Alexander dual ideal of in $(\mathcal{K})$ which comes from several intersections of ideals, we define the following notation.

Definition 4.1. If we have $(1,1)>(1,2)>\cdots>(1, n)>(2,1)>$ $(2,2)>\cdots>(2, n)>\cdots>(m, n-1)>(m, n)$, we write

$$
\left(a_{1}, a_{2}\right) \uplus 1=\left\{\begin{array}{ccc}
\left(a_{1}, a_{2}+1\right) & \text { if } & a_{2}<n \\
\left(a_{1}+1,1\right) & \text { if } & a_{2}=n
\end{array},\right.
$$

and similarly,

$$
\left(a_{1}, a_{2}\right) \uplus 2=\left\{\begin{array}{ccc}
\left(a_{1}, a_{2}+2\right) & \text { if } & a_{2}<n-1 \\
\left(a_{1}+1,1\right) & \text { if } & a_{2}=n-1 \\
\left(a_{1}+1,2\right) & \text { if } & a_{2}=n .
\end{array}\right.
$$

We are now ready to show $(\text { in }(\mathcal{K}))^{*}$ is generated in the same degree $d$ and $\operatorname{reg}(\text { in }(\mathcal{K}))^{*}=d$ as the main lemma of this paper.

Lemma 4.2. The Alexander dual of in $(\mathcal{K})$, $(\text { in }(\mathcal{K}))^{*}$, is generated by square-free monomials with degree $m n-1+t_{2}-\left(s_{2}-1\right)+t_{1}-\left(s_{1}-1\right)$ and $\operatorname{reg}(\operatorname{in}(\mathcal{K}))^{*}=m n-1+t_{2}-\left(s_{2}-1\right)+t_{1}-\left(s_{1}-1\right)$.

Proof. We prove this lemma by inducting on $n$. Since the proof is long, we state the steps of the proof here. We will show the case of $m=n$ first. We list the Alexander dual ideal then use the same technique as Lemma 3.5 to show it has the right regularity. Then assume the statement is true for the case of $m \times(n-1)$ size matrix and find a filtration starting from the Alexander dual of the case $m \times n$ ending at the Alexander dual of the case $m \times(n-1)$. Using the fact that this filtration has good quotients, we show the Alexander dual has the right regularity. One key point is to find the filtration; 
therefore, the proof will emphasize picking the right filtration. We may assume $s_{1} \geq s_{2}$; then the generators of the ideal $(\text { in }(\mathcal{K}))^{*}$ do not involve variables $x_{i, j}, y_{l, k}, z_{p, q}$ when $i, l, p>s_{1}$. Hence, we can further assume $s_{1}=m$.

When $m=n$, we have $m=s_{1} \leq t_{1} \leq m$. We list the generators of $(\text { in }(\mathcal{K}))^{*}$ here. It is easy to see there is a pattern, and it makes sense to find a filtration of (in $(\mathcal{K}))^{*}$. Let $B=\left(B_{2}, B_{3}, \ldots, B_{s_{2}}\right)$ be such that $0 \leq$ $B_{s_{2}}<\cdots<B_{2}<t_{2}$, and write $\underline{y}_{B}=\prod_{b_{1}=B_{2}+2}^{t_{2}} y_{1, b_{1}} \cdots \prod_{b_{s_{2}}=1}^{B_{s_{2}}} y_{s_{2}, b_{s_{2}}}$. Then

$$
\begin{aligned}
& (\operatorname{in}(\mathcal{K}))^{*}=\left(\left\{\prod_{(i, j)=(1,1)}^{\left(u_{1}, u_{2}\right)} z_{i, j} \prod_{(l, k)=\left(u_{1}, u_{2}\right) \uplus 2}^{(m, n)} x_{l, k} \underline{y}_{B} x_{q, m-q+1} \mid\right.\right. \\
& \left.\left(u_{1}, u_{2}\right)<(m-1, m-1), 1 \leq q \leq m\right\}, \\
& \left\{\prod_{(i, j)=(1,1)}^{\left(u_{1}, u_{2}\right)} z_{i, j} \prod_{(l, k)=\left(u_{1}, u_{2}\right) \uplus 2}^{(m, n)} x_{l, k} \underline{y}_{B} x_{q, m-q+1} \mid\right. \\
& \left.(m-1, m-1) \leq\left(u_{1}, u_{2}\right) \leq(m-1,1), 1 \leq q \leq m-1\right\}, \\
& \left\{\prod_{(i, j)=(1,1)}^{\left(u_{1}, u_{2}\right)} z_{i, j} \prod_{(l, k)=\left(u_{1}, u_{2}\right) \uplus 2}^{(m, n)} x_{l, k} \underline{y}_{B} y_{m, 1} \mid\right. \\
& \left.(m-1, m-1) \leq\left(u_{1}, u_{2}\right) \leq(m-1,1), s_{2}<m\right\}, \\
& \left\{\prod_{(i, j)=(1,1)}^{\left(u_{1}, u_{2}\right)} z_{i, j} \prod_{(l, k)=\left(u_{1}, u_{2}\right) \uplus 2}^{(m, n)} x_{l, k} \underline{y}_{B} y_{m, 2} \mid\right. \\
& \left.(m-1, m-1) \leq\left(u_{1}, u_{2}\right) \leq(m-1,1), s_{2}=m\right\}, \\
& \left\{\prod_{(i, j)=(1,1)}^{\left(u_{1}, u_{2}\right)} z_{i, j} \prod_{(l, k)=\left(u_{1}, u_{2}\right) \uplus 2}^{(m, n)} x_{l, k} \underline{y}_{B} x_{q, m-q+1} \mid\right. \\
& (p+1, m-p+2) \leq\left(u_{1}, u_{2}\right) \leq(p, m-p), \\
& 1 \leq q \leq p \leq m-2\} \text {, }
\end{aligned}
$$




$$
\begin{aligned}
& \left\{\prod_{(i, j)=(1,1)}^{\left(u_{1}, u_{2}\right)} z_{i, j} \prod_{(l, k)=\left(u_{1}, u_{2}\right) \uplus 2}^{(m, n)} x_{l, k} \underline{y}_{B} x_{\left(u_{1}, u_{2}\right) \uplus 1} \mid\right. \\
& (p+1, m-p+2) \leq\left(u_{1}, u_{2}\right) \leq(p, m-p) \text {, } \\
& 1 \leq p \leq m-2\} \text {, } \\
& \left\{\prod_{(i, j)=(1,1)}^{\left(u_{1}, u_{2}\right)} z_{i, j} \prod_{(l, k)=\left(u_{1}, u_{2}\right) \uplus 2}^{(m, n)} x_{l, k} \underline{y}_{B} y_{m-q+1, q} \mid\right. \\
& (p+1, m-p+2) \leq\left(u_{1}, u_{2}\right) \leq(p, m-p) \text {, } \\
& \left.1 \leq p \leq m-2,1 \leq q \leq m-p, s_{2}<m\right\} \text {, } \\
& \left\{\prod_{(i, j)=(1,1)}^{\left(u_{1}, u_{2}\right)} z_{i, j} \prod_{(l, k)=\left(u_{1}, u_{2}\right) \uplus 2}^{(m, n)} x_{l, k} \underline{y}_{B} y_{m-q+1, q+1} \mid\right. \\
& (p+1, m-p+2) \leq\left(u_{1}, u_{2}\right) \leq(p, m-p) \text {, } \\
& \left.\left.1 \leq p \leq m-2,1 \leq q \leq m-p, s_{2}=m\right\}\right)
\end{aligned}
$$

Notice that all the elements are in the same degree $m m-1+1+t_{2}-$ $\left(s_{2}-1\right)$. We find a filtration starting from $(\text { in }(\mathcal{K}))^{*}$ and ending at $\left(x_{m, 1}, x_{m-1,2}, \ldots, x_{1, m}\right)$. Each quotient of this filtration will have the form $P / P L$, where $P$ is an ideal generated in the same degree and $L$ is an ideal generated by variables such that those variables form a regular sequence modulo $P$. Then we use the same technique in the Lemma 3.5 to find the regularity of the Alexander dual ideal.

We look at the variable $y_{s_{2}, 1}$. The elements in $(\text { in }(\mathcal{K}))^{*}$ that are divisible by $y_{s_{2}, 1}$ must have $B_{s_{2}}>0$. The elements in $(\text { in }(\mathcal{K}))^{*}$ that are not divisible by $y_{s_{2}, 1}$ must have $B_{s_{2}}=0$. Hence $\left(\right.$ in $\left.(\mathcal{K})^{*}\right)=$ $\left(A_{1}\left(y_{s_{2}, 1}, \ldots, y_{s_{2}-1,2}, \cdots y_{1, s_{2}}\right), C_{1} y_{s_{2}, 1}\right)$, where the elements in $C_{1}$ have $B_{s_{2}}>1$. Also, all the elements of $A_{1}$ and $C_{1}$ are in the same degree. Furthermore, $A_{1} \cap C_{1}=C_{1}\left(\left\{a_{1, i}\right\}\right),\left(y_{s_{2}, 1}, \ldots, y_{1, s_{2}}\right)$ is a regular sequence modulo $A_{1}$ and $\left(y_{s_{2}, 1},\left\{a_{1, i}\right\}\right)$ is a regular sequence modulo $C_{1}$. We look at the following filtration

$$
(\operatorname{in}(\mathcal{K}))^{*} \subset\left(A_{1}, C_{1} y_{s_{2}, 1}\right) \subset\left(A_{1}, C_{1}\right) .
$$

Using the proof of Lemma 3.5, one can see that the quotients are: 
$A_{1} / A_{1}\left(y_{s_{2}, 1}, \ldots, y_{1, s_{2}}\right)$ and $C_{1} / C_{1}\left(\left\{a_{1, i}\right\}, y_{s_{2}, 1}\right)$. Notice the following equalities: $\operatorname{reg}\left(A_{1} / A_{1}\left(y_{s_{2}, 1}, \ldots, y_{1, s_{2}}\right)\right)=\operatorname{reg}\left(A_{1}\right)$ and $\operatorname{reg}\left(C_{1} / C_{1}\left(\left\{a_{1, i}\right\}\right.\right.$, $\left.y_{s_{2}, 1}\right)=\operatorname{reg}\left(C_{1}\right)$.

Next, we look at $y_{s_{2}, 2}$ and write

$$
\left(A_{1}, C_{1}\right)=\left(A_{2}\left(y_{s_{2}, 2}, \ldots, y_{1, s_{2}+1}\right), C_{2} y_{s_{2}, 2}\right) .
$$

As before, we have the filtration

$$
\left(A_{1}, C_{1}\right) \subset\left(A_{2}, C_{2} y_{s_{2}, 2}\right) \subset\left(A_{2}, C_{2}\right),
$$

and the quotients are $A_{2} / A_{2}\left(y_{s_{2}, 2}, \ldots, y_{1, s_{2}+1}\right)$ and $C_{2} / C_{2}\left(\left\{a_{2, i}\right\}, y_{s_{2}, 2}\right)$. We can continue with $y_{s_{2}, 3} \ldots$ until $y_{s_{2}, t_{2}-\left(s_{2}-1\right)}$; we will reduce $(\text { in }(\mathcal{K}))^{*}$ to an ideal $J_{1}$ which is generated in the same degree $m m-1+1$.

Now we look at the variable $z_{1,1}$. When an element has $\left(u_{1}, u_{2}\right)=$ $(0,0), z_{1,1}$ is not a factor of this element. When $\left(u_{1}, u_{2}\right) \leq(1,1)$, then $z_{1,1}$ is a factor. The ideal $J_{1}$ can be written as $\left(D_{1,1}\left(z_{1,1},\left\{d_{1,1, i}\right\}\right), E_{1,1} z_{1,1}\right)$ where $\left\{d_{1,1, i}\right\}$ is a set of variables and is a regular sequence modulo $D_{1,1}$. Hence, we have the filtration

$$
J_{1} \subset\left(D_{1,1}, E_{1,1} z_{1,1}\right) \subset\left(D_{1,1}, E_{1,1}\right)
$$

with quotients $D_{1,1} / D_{1,1}\left(z_{1,1},\left\{d_{1,1, i}\right\}\right)$ and $E_{1,1} / E_{1,1}\left(\left\{e_{1,1, i}\right\}, z_{1,1}\right)$. We look at $z_{1,2}$ next and reduce $J_{1}$ to an ideal $\left(D_{1,2}, E_{1,2}\right)$; we continue with $z_{m, m-1}$. We can find a filtration from $J_{1}$ to $\left(D_{m, m-1}, E_{m, m-1}\right)=$ $\left(x_{m, 1}, \ldots, x_{1 m}\right)$.

Since reg $\left(x_{m, 1}, \ldots, x_{1, m}\right)=\operatorname{reg}\left(D_{m, m-1}, E_{m, m-1}\right)=1$, it follows that $\operatorname{reg}\left(D_{m, m-1}\right)$ and $\operatorname{reg}\left(E_{m, m-1}\right)$ are equal to 1 . Also, the final quotient

$$
\left(D_{m, m-1}, E_{m, m-1}\right) /\left(D_{m, m-1}, E_{m, m-1} z_{m, m-1}\right)
$$

has regularity equal to the regularity of $E_{m, m-1}$. It follows that

$$
\operatorname{reg}\left(D_{m, m-1}, E_{m, m-1} z_{m, m-1}\right)=2 .
$$

Since the quotient $\left(D_{m, m-1}, E_{m, m-1} z_{m, m-1}\right) /\left(D_{m, m-2}, E_{m, m-2}\right)$ has regularity equal to the regularity of $D_{m, m-1}$, which is 1 , we have $\operatorname{reg}\left(D_{m, m-2}, E_{m, m-2}\right)=2$. Continuing the same argument, we obtain $\operatorname{reg}\left(J_{1}\right)=1+m m-1$. Hence, $\operatorname{reg}\left((\operatorname{in} \mathcal{K})^{*}\right)=1+m m-1+t_{2}-\left(s_{2}-1\right)$. This finishes the case of an $m \times m$ size matrix. 
For the induction steps, we write $I_{n}$ for the Alexander dual ideal $(\text { in }(\mathcal{K}))^{*}$ in the $m \times n$ matrix case. We assume, by induction hypothesis, that reg $\left(I_{n-1}\right)=\operatorname{deg}\left(I_{n-1}\right)=m(n-1)-1+t_{1}-\left(s_{1}-1\right)+t_{2}-\left(s_{2}-1\right)$. We will show that we can build a filtration from $I_{n}$ to $I_{n-1}$ such that the quotients are in the form $P / P L$ where $P$ is an ideal and $L$ is an ideal generated by variables such that they are a regular sequence modulo $P$.

We know that each summand of in $(\mathcal{K})$ is generated by monomials satisfying the assumption of Lemma 3.2. For a fixed variable $y_{i, j}$, we can write $\left(h_{Y}\right)^{*}=\left(A^{Y} y_{i, j}, A^{Y}\left(\left\{a_{l}^{Y}\right\}\right), B^{Y} y_{i, j}\right)$. Also, $A^{Y} \cap B^{Y}=B^{Y}\left(\left\{b_{i}^{Y}\right\}\right)$, where $\left\{b_{i}^{Y}\right\}$ are variables such that $\left(y_{i, j},\left\{b_{i}^{Y}\right\}\right)$ is a regular sequence modulo $B^{Y}$ and $\left(y_{i, j},\left\{a_{l}^{Y}\right\}\right)$ is a regular sequence modulo $A^{Y}$. Similarly, $\left(h_{U}\right)^{*}=\left(A^{U} y_{i j}, A^{U}\left(\left\{a_{l}^{U}\right\}\right), B^{U} y_{i j}\right)$, $\left(h_{W}\right)^{*}=\left(A^{W} y_{i, j}, A^{W}\left(\left\{a_{l}^{W}\right\}\right), B^{W} y_{i, j}\right)$, and all other components of (in $(\mathcal{K}))^{*}$ involving $y_{i, j}$. For $\left(h_{X}\right)^{*}$ that does not involve $y_{i, j}$, we leave as it is and similarly for others that do not involve $y_{i, j}$.

Claim. Let $I_{y_{i, j}}$ be the ideal coming from (in $\left.(K)\right)^{*}$ deleting the variable $y_{i j}$. Then $I_{y_{i, j}}$ contains $(\text { in }(K))^{*}$. Assume $\operatorname{reg}\left(I_{y_{i, j}}\right)=$ $\operatorname{deg}\left(I_{y_{i, j}}\right)$. Then there is a filtration from in $(\mathcal{K})^{*}$ to the ideal

$$
I_{y_{i, j}}=\left(h_{X}\right)^{*} \cap\left(A^{Y}, B^{Y}\right) \cap\left(h_{g}\right)^{*} \cap\left(A^{U}, B^{U}\right) \cap \cdots \cap\left(A^{W}, B^{W}\right)
$$

such that the quotients are the form $P / P L$ where $P$ is an ideal and $L$ is an ideal generated by variables such that they are a regular sequence modulo $P$. Moreover, the relation of the regularity is $\operatorname{reg}\left(\right.$ in $\left.(\mathcal{K})^{*}\right)=$ $\operatorname{reg}\left(I_{y_{i, j}}\right)+1=\operatorname{deg}\left(I_{y_{i, j}}\right)+1$.

With this claim, we can continue picking another variable and reduce $(\text { in }(\mathcal{K}))^{*}$ to a bigger ideal that does not involve the new variable. By picking the right variables at each step, we reach an ideal that does not involve any $z_{i, n}, x_{i, n}$ or $y_{i, n}$. This ideal is $I_{n-1}$, and we can use induction to show the lemma. We prove this claim first then find the right variables such that the filtration has quotients we need.

Proof of the claim. Without loss of generality, we just need to show that there is a filtration from $\left(A^{Y}\left(y_{i, j},\left\{a_{l}^{Y}\right\}\right), B^{Y} y_{i, j}\right) \cap$ $\left.\left(A^{U}\left(y_{i, j},\left\{a_{l}^{U}\right\}\right), B^{U} y_{i, j}\right)\right)$ to $\left(A^{Y}, B^{Y}\right) \cap\left(A^{U}, B^{U}\right)$ with quotients in the form $P / P L$ as above. For convenience, we write $A^{Y}\left(\left\{a_{l}^{Y}\right\}\right)=A^{Y} C$ 
and $A^{U}\left(\left\{a_{l}^{U}\right\}\right)=A^{U} D$. Consider the following filtration:

$$
\begin{aligned}
& \left(A^{Y} y_{i, j}, A^{Y} C, B^{Y} y_{i, j}\right) \cap\left(A^{U} y_{i, j}, A^{U} D, B^{U} y_{i, j}\right)=: J_{0} \\
& \subset\left(\left(A^{Y} \cap A^{U}\right) y_{i, j},\left(A^{Y} \cap A^{U}\right) C,\left(B^{Y} \cap A^{U}\right) y_{i, j},\left(A^{Y} \cap B^{U}\right) y_{i, j},\right. \\
& \left.\left(B^{Y} \cap B^{U}\right) y_{i, j}\right)=: J_{1} \\
& \subset\left(\left(A^{Y} \cap A^{U}\right),\left(B^{Y} \cap A^{U}\right) y_{i, j},\left(A^{Y} \cap B^{U}\right) y_{i, j},\left(B^{Y} \cap B^{U}\right) y_{i, j}\right)=: J_{2} \\
& \subset\left(\left(A^{Y} \cap A^{U}\right),\left(B^{Y} \cap A^{U}\right),\left(A^{Y} \cap B^{U}\right) y_{i, j},\left(B^{Y} \cap B^{U}\right) y_{i, j}\right)=: J_{3} \\
& \subset\left(\left(A^{Y} \cap A^{U}\right),\left(B^{Y} \cap A^{U}\right),\left(A^{Y} \cap B^{U}\right),\left(B^{Y} \cap B^{U}\right) y_{i, j}\right)=: J_{4} \\
& \subset\left(\left(A^{Y} \cap A^{U}\right),\left(B^{Y} \cap A^{U}\right),\left(A^{Y} \cap B^{U}\right),\left(B^{Y} \cap B^{U}\right)\right) \\
& =\left(A^{Y}, B^{Y}\right) \cap\left(A^{U}, B^{U}\right)=: J_{5} .
\end{aligned}
$$

The quotient

$$
\begin{aligned}
J_{1} / J_{0}= & \left(A^{Y} \cap A^{U}\right) C /\left(\left(A^{Y} \cap A^{U}\right) C\left(D, y_{i, j}\right),\left(A^{Y} \cap B^{Y}\right)\right. \\
\cap & A^{U} C y_{i, j},\left(A^{Y} \cap A^{U} \cap B^{U}\right) C y_{i, j}, \\
& \left.\left(A^{Y} \cap B^{Y}\right) \cap\left(A^{U} \cap B^{U}\right) C y_{i, j}\right) \\
= & \left(A^{Y} \cap A^{U}\right) C /\left(\left(A^{Y} \cap A^{U}\right) C\left(D, y_{i, j}\right)\right) .
\end{aligned}
$$

Similarly, $J_{l+1} / J_{l}$ has the form $P_{l} / P_{l} L_{l}$ with $L_{l}$ variables that form a regular sequence modulo $P_{l}$ for $l=1,2,3,4$. For $l=1$,

$$
\begin{aligned}
J_{2} / J_{1}= & \left(A^{Y} \cap A^{U}\right) /\left(\left(A^{Y} \cap A^{U}\right)\left(C, y_{i j}\right),\left(A^{Y} \cap B^{Y} \cap A^{U}\right) y_{i j},\right. \\
& \left(A^{Y} \cap A^{U} \cap B^{U}\right) y_{i j}, \\
& \left.\left(A^{Y} \cap B^{Y}\right) \cap\left(A^{U} \cap B^{U}\right) y_{i j}\right) \\
= & \left(A^{Y} \cap A^{U}\right) /\left(\left(A^{Y} \cap A^{U}\right)\left(C, y_{i j}\right)\right) .
\end{aligned}
$$

Hence, $\operatorname{reg}\left(J_{2} / J_{1}\right)=\operatorname{reg}\left(A^{Y} \cap A^{U}\right)$. For $l=2$,

$$
\begin{aligned}
J_{3} / J_{2}= & \left(B^{Y} \cap A^{U}\right) /\left(\left(B^{Y} \cap A^{U}\right) y_{i j},\left(B^{Y} \cap A^{Y} \cap A^{U}\right),\right. \\
& \left(B^{Y} \cap A^{Y}\right) \cap\left(A^{U} \cap B^{U}\right) y_{i j}, \\
& \left.\left(B^{Y} \cap A^{U} \cap B^{U}\right) y_{i j}\right) \\
= & \left(B^{Y} \cap A^{U}\right) /\left(\left(B^{Y} \cap A^{U}\right)\left(\left\{b_{i}^{Y}\right\}, y_{i j}\right)\right) .
\end{aligned}
$$

Thus, $\operatorname{reg}\left(J_{3} / J_{2}\right)=\operatorname{reg}\left(B^{Y} \cap A^{U}\right)$. For $l=3$,

$$
J_{4} / J_{3}=\left(A^{Y} \cap B^{U}\right) /\left(\left(A^{Y} \cap B^{U}\right)\left(y_{i j},\left\{b_{i}^{Y}\right\}\right)\right) .
$$


Therefore, $\operatorname{reg}\left(J_{4} / J_{3}\right)=\operatorname{reg}\left(A^{Y} \cap B^{U}\right)$. Finally, for $l=4$,

$$
J_{5} / J_{4}=\left(B^{Y} \cap B^{U}\right) /\left(\left(B^{Y} \cap B^{U}\right)\left(y_{i j},\left\{b_{i}^{Y}\right\},\left\{b_{i}^{U}\right\}\right) .\right.
$$

We obtain $\operatorname{reg}\left(J_{5} / J_{4}\right)=\operatorname{reg}\left(B^{Y} \cap B^{U}\right)$. By assumption,

$$
\operatorname{reg}\left(A^{Y}, B^{Y}\right) \cap\left(A^{U}, B^{U}\right)=d=\operatorname{deg}\left(A^{Y}, B^{Y}\right) \cap\left(A^{U}, B^{U}\right),
$$

it follows that

$$
\begin{aligned}
\operatorname{deg}\left(A^{Y} \cap A^{U}\right) & =\operatorname{deg}\left(B^{Y} \cap A^{U}\right) \\
& =\operatorname{deg}\left(A^{Y} \cap B^{U}\right)=\operatorname{deg}\left(B^{Y} \cap B^{U}\right)=d
\end{aligned}
$$

and

$$
\begin{aligned}
\operatorname{reg}\left(A^{Y} \cap A^{U}\right) & =\operatorname{reg}\left(B^{Y} \cap A^{U}\right) \\
& =\operatorname{reg}\left(A^{Y} \cap B^{U}\right)=\operatorname{reg}\left(B^{Y} \cap B^{U}\right)=d .
\end{aligned}
$$

Also notice that $\operatorname{reg}\left(A^{Y} \cap A^{U}\right) C \geq \operatorname{deg}\left(A^{Y} \cap A^{U}\right) C \geq d+1$. We use the regularity of the quotients of the filtration to obtain the regularity of $J_{0}$. From the short exact sequence of regularity, we obtain reg $J_{4}=$ $\operatorname{reg}\left(J_{5}\right)+1=d+1$ and $\operatorname{reg} J_{3}=d+1=\operatorname{reg} J_{2}=d+1=\operatorname{reg} J_{1}=d+1$. Notice $\operatorname{reg} J_{0} \geq \operatorname{deg} J_{0} \geq \operatorname{deg} J_{1}+1=d+1$. Assume reg $J_{0} \geq d+2$. Then $\operatorname{reg} J_{1}=\max \left\{\operatorname{reg} J_{0}, \operatorname{reg} J_{1} / J_{0}=\operatorname{reg}\left(A^{Y} \cap A^{U}\right) C\right\} \geq \bar{d}+2$, a contradiction. Hence reg $J_{0}=\operatorname{deg} J_{0}=d+1$. This completes the proof of the claim.

We now focus on finding the right variables for the filtration. Since $s_{1} \geq s_{2}$, we assume $s_{1}=m$ and observe that $(\text { in }(\mathcal{K}))^{*}$ does not involve $z_{m, n}$. If $t_{2}=n$, by using the claim above, we can find a filtration starting from $(\text { in }(\mathcal{K}))^{*}$ to an ideal $J_{y_{1, n}}$, where $y_{1, n}$ is not a factor of the minimal monomial generators of $J_{y_{1 n}}$; otherwise, we find a filtration to an ideal $J_{y_{2 n}}$ such that $y_{2, n}$ is not a factor of the minimal monomial generators of $J_{y_{2, n}}$. The next step is to look at $z_{m-1, n}$ and find a filtration until an ideal $J_{z_{m-1, n}}$ such that $z_{m-1, n}$ is not a factor of $J_{z_{m-1, n}}$. Next we consider $z_{m-2, n}$ and continue to $z_{m-3, n} \ldots$ until $z_{1 n}$. Finally, we consider $x_{1, n}$. Then we will get the ideal $I_{n-1}$ if $t_{2}=n$; otherwise, we continue with $x_{2, n}$ and get the ideal $I_{n-1}$. We will have a filtration as the following:

$$
I_{n} \subset J_{y_{1, n}} \subset J_{y_{2, n}} \subset J_{z_{m-1, n}} \subset J_{z_{m-2, n}} \subset \cdots \subset J_{z_{1, n}} \subset J_{x_{1, n}}=I_{n-1}
$$


or

$$
I_{n} \subset J_{y_{2, n}} \subset J_{z_{m-1, n}} \subset J_{z_{m-2, n}} \subset \cdots \subset J_{z_{1, n}} \subset J_{x_{1, n}} \subset J_{x_{2, n}}=I_{n-1} .
$$

Assume that $\left\{J_{l}\right\}_{l=1}^{k_{1}}$ is the sub-filtration from $J_{k_{1}}=I_{n-1}$ to $J_{x_{1}, n}=J_{1}$ and $J_{l+1} / J_{l}$ has the form $P_{l} / P_{l} L_{l}$ with $L_{l}$ variables that form a regular sequence modulo $P_{l}$ for all $l$. Hence, $\operatorname{reg}\left(J_{l+1} / J_{l}\right)=\operatorname{reg} P_{l}$ for all $l=1, \ldots, k_{1}-1$. In particular, we obtain $\operatorname{reg}\left(I_{n-1} / J_{k_{1}-1}\right)=$ reg $\left(I_{n-1}\right)=\operatorname{deg}\left(I_{n-1}\right)$ where the last equality coming from the induction hypothesis. It follows that

$$
\operatorname{reg}\left(P_{l}\right)=\operatorname{deg}\left(P_{l}\right)=\operatorname{reg}\left(I_{n-1}\right)
$$

for all $l$.

By using a similar argument as in the proof of the claim, we have $\operatorname{reg}\left(J_{x_{1}, n}\right)=\operatorname{deg}\left(J_{x_{1}, n}\right)=\operatorname{reg}\left(I_{n-1}\right)+1$. By induction again, we obtain

$$
\begin{aligned}
\operatorname{reg} I_{n} & =\operatorname{reg} I_{n-1}+2+m-1+1 \\
& =m(n-1)-1+t_{1}-1-\left(s_{1}-1\right)+t_{2}-1-\left(s_{2}-1\right)+m+2 \\
& =m n-1+t_{1}-\left(s_{1}-1\right)+t_{2}-\left(s_{2}-1\right) \\
& =\operatorname{deg} I_{n-1}+2+m-1+1 \\
& =\operatorname{deg} I_{n} .
\end{aligned}
$$

The proof of Lemma 4.2 is now complete.

We are now ready to prove Theorem 1.1.

Proof of Theorem 1.1. We know that $k[X, Y, Z] /($ in $(\mathcal{K}))$ is CohenMacaulay by Lemma 4.2 and Theorem 1.3. Hence, $\mathcal{R}(\mathbf{D})=k[X, Y, Z] / \mathcal{K}$ is Cohen-Macaulay [4].

The following example is computed by [6], and it shows how the filtration of the Alexander dual ideal looks.

Example 4.3. Let $X, Y, Z$ be a $2 \times 3$ matrices, $X_{2,3}, Y_{2,2}$ are $2 \times 3$ and $2 \times 2$ submatrices of $X$ and $Y$, and let $\mathcal{K}$ be the defining ideal of the $\mathcal{R}(\mathbf{D})$ in the ring $k[X, Y, Z]$. The initial ideal of $\mathcal{K}$ via the lexicographic order with $z_{i, j}>x_{l, k}>y_{p, q}$ for any $i, j, l, k, p, q, z_{i, j}>z_{l, k}$ if $i<l$ or 
$i=l$ and $j<k$, and $x_{i, j}>x_{l, k}$ and $y_{i, j}>y_{l, k}$ if $i<l$ or $i=l$ and $j>k$ is generated by

$$
\begin{aligned}
& \left\{z_{22} x_{23}, z_{21} x_{22}, z_{21} x_{23}, z_{21} x_{13} y_{22}, z_{13} x_{21}, z_{13} x_{22}, z_{13} x_{23},\right. \\
& z_{13} x_{12} y_{21}, z_{13} x_{12} y_{11} y_{22}, z_{13} x_{12} y_{11} y_{23}, z_{13} x_{13} y_{21}, \\
& z_{13} x_{13} y_{22}, z_{12} x_{21}, z_{12} x_{22}, z_{12} x_{23}, z_{12} x_{11} y_{23}, z_{12} x_{12} y_{21}, \\
& z_{12} x_{12} y_{23}, z_{12} x_{12} y_{11} y_{22}, z_{12} x_{13}, z_{11} y_{22}, z_{11} y_{12} y_{23}, \\
& z_{11} x_{21}, z_{11} x_{22}, z_{11} x_{23}, z_{11} x_{11} y_{23}, z_{11} x_{12}, z_{11} x_{13}, \\
& \left.y_{12} y_{21}, x_{12} x_{21}, x_{13} x_{21}, x_{13} x_{22}\right\}
\end{aligned}
$$

The Alexander dual ideal is $I_{3}$, and $I_{3}$ is minimally generated by square-free monomials in degree 8 . The generators are:

$$
\begin{aligned}
& \left\{z_{11} z_{12} z_{13} z_{21} z_{22} x_{13} x_{12} y_{12}, z_{11} z_{12} z_{13} z_{21} x_{13} x_{12} x_{23} y_{12}\right. \text {, } \\
& z_{11} z_{12} z_{13} x_{13} x_{12} x_{23} x_{22} y_{12}, z_{11} z_{12} z_{13} z_{21} z_{22} x_{13} x_{21} y_{12} \text {, } \\
& z_{11} z_{12} z_{13} z_{21} x_{13} x_{23} x_{21} y_{12}, z_{11} z_{12} z_{13} z_{21} z_{22} x_{22} x_{21} y_{12} \text {, } \\
& z_{11} z_{12} z_{13} z_{21} x_{23} x_{22} x_{21} y_{12}, z_{11} z_{12} z_{13} x_{13} x_{23} x_{22} x_{21} y_{12} \text {, } \\
& z_{11} z_{12} x_{13} x_{12} x_{23} x_{22} x_{21} y_{12}, z_{11} x_{13} x_{12} x_{11} x_{23} x_{22} x_{21} y_{12} \text {, } \\
& z_{11} x_{13} x_{12} x_{23} x_{22} x_{21} y_{12} y_{23}, z_{11} z_{12} z_{13} x_{23} x_{22} x_{21} y_{12} y_{22} \text {, } \\
& x_{13} x_{12} x_{11} x_{23} x_{22} x_{21} y_{12} y_{22}, x_{13} x_{12} x_{23} x_{22} x_{21} y_{12} y_{23} y_{22} \text {, } \\
& z_{11} z_{12} z_{13} z_{21} z_{22} x_{13} x_{12} y_{21}, z_{11} z_{12} z_{13} z_{21} x_{13} x_{12} x_{23} y_{21} \text {, } \\
& z_{11} z_{12} z_{13} x_{13} x_{12} x_{23} x_{22} y_{21},, z_{11} z_{12} z_{13} z_{21} z_{22} x_{13} x_{21} y_{21} \text {, } \\
& z_{11} z_{12} z_{13} z_{21} x_{13} x_{23} x_{21} y_{21}, z_{11} z_{12} z_{13} z_{21} z_{22} x_{22} x_{21} y_{21} \text {, } \\
& z_{11} z_{12} z_{13} z_{21} x_{23} x_{22} x_{21} y_{21}, z_{11} z_{12} z_{13} x_{13} x_{23} x_{22} x_{21} y_{21} \text {, } \\
& z_{11} z_{12} x_{13} x_{12} x_{23} x_{22} x_{21} y_{21}, z_{11} x_{13} x_{12} x_{11} x_{23} x_{22} x_{21} y_{21} \text {, } \\
& z_{11} z_{12} x_{13} x_{23} x_{22} x_{21} y_{11} y_{21}, z_{11} x_{13} x_{12} x_{23} x_{22} x_{21} y_{23} y_{21} \text {, } \\
& z_{11} x_{13} x_{23} x_{22} x_{21} y_{11} y_{23} y_{21}, z_{11} z_{12} z_{13} x_{23} x_{22} x_{21} y_{22} y_{21} \text {, } \\
& z_{11} z_{12} x_{12} x_{23} x_{22} x_{21} y_{22} y_{21}, z_{11} z_{12} x_{23} x_{22} x_{21} y_{11} y_{22} y_{21} \text {, } \\
& z_{11} z_{12} x_{23} x_{22} x_{21} y_{23} y_{22} y_{21}, z_{11} x_{13} x_{23} x_{22} x_{21} y_{23} y_{22} y_{21} \text {, } \\
& \left.x_{13} x_{12} x_{23} x_{22} x_{21} y_{23} y_{22} y_{21}\right\} \text {. }
\end{aligned}
$$

The filtration to $I_{2}$, the Alexander dual ideal in the $2 \times 2$ matrix case is listed here.

$$
J_{y_{23}}=\left(x_{13} x_{12} x_{23} x_{22} x_{21} y_{22} y_{21}, x_{13} x_{12} x_{23} x_{22} x_{21} y_{12} y_{22},\right.
$$


$z_{11} x_{13} x_{23} x_{22} x_{21} y_{22} y_{21}, z_{11} x_{13} x_{23} x_{22} x_{21} y_{11} y_{21}$, $z_{11} x_{13} x_{11} x_{23} x_{22} x_{21} y_{21}, z_{11} x_{13} x_{12} x_{23} x_{22} x_{21} y_{12}$, $z_{11} z_{12} x_{23} x_{22} x_{21} y_{22} y_{21}, z_{11} z_{12} z_{13} x_{23} x_{22} x_{21} y_{12} y_{22}$, $z_{11} z_{12} z_{12} x_{13} x_{23} x_{22} x_{21} y_{21}, z_{11} z_{12} z_{13} x_{13} x_{23} x_{22} x_{21} y_{12}$, $z_{11} z_{12} z_{13} x_{13} x_{12} x_{23} x_{22} y_{21}, z_{11} z_{12} z_{13} x_{13} x_{12} x_{23} x_{22} y_{12}$, $z_{11} z_{12} z_{13} z_{21} x_{23} x_{22} x_{21} y_{21}, z_{11} z_{12} z_{13} z_{21} x_{23} x_{22} x_{21} y_{11}$, $z_{11} z_{12} z_{13} z_{21} x_{13} x_{23} x_{21} y_{21}, z_{11} z_{12} z_{13} z_{21} x_{13} x_{23} x_{21} y_{12}$, $z_{11} z_{11} z_{13} z_{21} x_{13} x_{12} x_{23} y_{21}, z_{11} z_{11} z_{13} z_{21} x_{13} x_{12} x_{23} y_{12}$, $z_{11} z_{12} z_{13} z_{21} z_{22} x_{22} x_{21} y_{21}, z_{11} z_{11} z_{13} z_{21} z_{22} x_{22} x_{21} y_{12}$, $z_{11} z_{12} z_{13} z_{21} z_{22} x_{13} x_{21} y_{21}, z_{11} z_{12} z_{13} z_{21} z_{22} x_{13} x_{21} y_{12}$, $\left.z_{11} z_{12} z_{13} z_{21} z_{22} x_{13} x_{12} y_{21}, z_{11} z_{12} z_{13} z_{21} z_{22} x_{13} x_{12} y_{12}\right)$.

$J_{z_{13}}=\left(x_{13} x_{12} x_{23} x_{22} x_{21} y_{22} y_{21}, x_{13} x_{12} x_{23} x_{22} x_{21} y_{12} y_{22}\right.$, $z_{11} x_{13} x_{23} x_{22} x_{21} y_{22} y_{21}, z_{11} x_{13} x_{23} x_{22} x_{21} y_{11} y_{21}$, $z_{11} x_{13} x_{12} x_{23} x_{22} x_{21} y_{21}, z_{11} x_{13} x_{12} x_{23} x_{22} x_{21} y_{12}$, $z_{11} z_{12} x_{23} x_{22} x_{21} y_{22} y_{21}, z_{11} z_{12} x_{23} x_{22} x_{21} y_{12} y_{22}$, $z_{11} z_{12} x_{13} x_{23} x_{22} x_{21} y_{21}, z_{11} z_{12} x_{13} x_{23} x_{22} x_{21} y_{12}$, $z_{11} z_{12} x_{13} x_{12} x_{23} x_{22} y_{21}, z_{11} z_{12} x_{13} x_{12} x_{23} x_{22} y_{12}$, $z_{11} z_{12} z_{21} x_{23} x_{22} x_{21} y_{21}, z_{11} z_{12} z_{21} x_{23} x_{22} x_{21} y_{12}$, $z_{11} z_{12} z_{21} x_{13} x_{23} x_{21} y_{21}, z_{11} z_{12} z_{21} x_{13} x_{23} x_{21} y_{12}$, $z_{11} z_{12} z_{21} x_{13} x_{12} x_{23} y_{21}, z_{11} z_{12} z_{21} x_{13} x_{12} x_{23} y_{12}$, $z_{11} z_{12} z_{21} z_{22} x_{22} x_{21} y_{21}, z_{11} z_{12} z_{21} z_{22} x_{22} x_{21} y_{12}$, $z_{11} z_{12} z_{21} z_{22} x_{13} x_{21} y_{21}, z_{11} z_{12} z_{21} z_{22} x_{13} x_{21} y_{12}$, $\left.z_{11} z_{12} z_{21} z_{22} x_{13} x_{11} y_{21}, z_{11} z_{12} z_{21} z_{22} x_{13} x_{12} y_{12}\right)$.

$J_{x_{13}}=\left(x_{12} x_{23} x_{22} x_{21} y_{22} y_{21}, x_{12} x_{23} x_{22} x_{21} y_{12} y_{22}, z_{11} x_{23} x_{22} x_{21} y_{22} y_{21}\right.$, $z_{11} x_{23} x_{22} x_{21} y_{11} y_{21}, z_{11} x_{12} x_{23} x_{22} x_{21} y_{21}, z_{11} x_{12} x_{23} x_{22} x_{21} y_{12}$, $z_{11} z_{12} x_{23} x_{22} x_{21} y_{21}, z_{11} z_{12} x_{23} x_{22} x_{21} y_{12}, z_{11} z_{12} x_{12} x_{23} x_{22} y_{21}$, $z_{11} z_{12} x_{12} x_{23} x_{22} y_{12}, z_{11} z_{12} z_{21} x_{23} x_{21} y_{21}, z_{11} z_{12} z_{21} x_{23} x_{21} y_{12}$, $z_{11} z_{12} z_{21} x_{12} x_{23} y_{21}, z_{11} z_{12} z_{21} x_{12} x_{23} y_{12}$, $z_{11} z_{12} z_{21} z_{22} x_{21} y_{21}, z_{11} z_{12} z_{21} z_{22} x_{21} y_{12}$, $\left.z_{11} z_{12} z_{21} z_{22} x_{12} y_{21}, z_{11} z_{12} z_{21} z_{22} x_{12} y_{12}\right)$. 


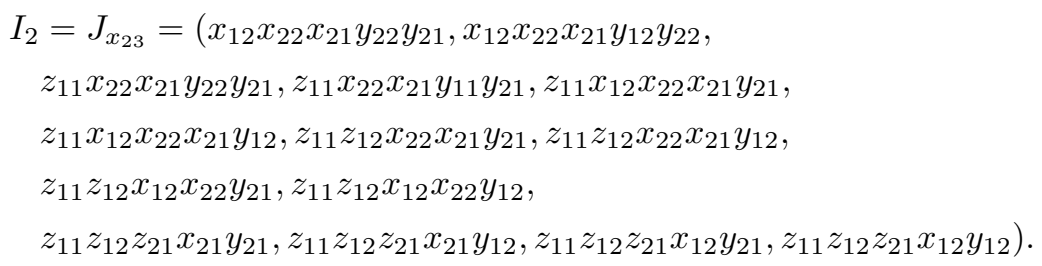

The filtration of $I_{2}$ is the following:

$$
\begin{gathered}
J_{y_{21}}=\left(x_{12} x_{22} x_{21} y_{22}, z_{11} x_{22} x_{21} y_{22}, z_{11} x_{22} x_{21} y_{11},\right. \\
z_{11} x_{12} x_{22} x_{21}, z_{11} z_{12} x_{22} x_{21}, z_{11} z_{12} x_{12} x_{22}, z_{11}, \\
\left.z_{12} z_{21} x_{21}, z_{11} z_{12} z_{21} x_{12}\right) . \\
J_{y_{22}}=\left(x_{12} x_{22} x_{21}, z_{11} x_{22} x_{21}, z_{11} z_{12} x_{12} x_{22},\right. \\
\left.z_{11} z_{12} z_{21} x_{21}, z_{11} z_{12} z_{21} x_{12}\right) . \\
J_{z_{11}}=\left(x_{22} x_{21}, z_{12} x_{12} x_{22}, z_{12} z_{21} x_{21}, z_{12} z_{21} x_{12}\right) . \\
J_{z_{12}}=\left(x_{22} x_{21}, x_{12} x_{22}, z_{21} x_{21}, z_{21} x_{12}\right), J_{z_{21}}=\left(x_{21}, x_{12}\right) .
\end{gathered}
$$

Acknowledgments. This work is based on the author's Ph.D. thesis from Purdue University under the direction of Professor Bernd Ulrich. The author is very grateful for so many useful suggestions from Professor Ulrich.

The author wishes to thank the referee for useful comments and suggestions.

\section{REFERENCES}

1. W. Bruns and J. Herzog, Cohen-Macaulay rings, Cambr. Stud. Adv. Math. 39, Cambridge University Press, Cambridge, 1993.

2. S.D. Cutkosky and T. Hà, Arithmetic Macaulayfication of projective schemes, J. Pure Appl. Alg. 201 (2005), 49-61.

3. J. Eagon and V. Reiner, Resolutions of Stanley-Reisner rings and Alexander duality, J. Pure Appl. Alg. 130 (1998), 165-175.

4. D. Eisenbud, Commutative algebra with a view toward algebraic geometry, Grad. Texts Math. 150, Springer-Verlag, New York, 1995.

5. S. Goto, Y. Nakamura and K. Nishida, Cohen-Macaulay graded rings associated to ideals, Amer. J. Math. 118 (1996), 1197-1213. 
6. D. Grayson and M. Stillman, Macaulay 2, A computer algebra system for computing in algebraic geometry and commutative algebra, available through anonymous ftp from http://www.math.uiuc.edu/Macaulay2.

7. J. Herzog, A. Simis and W. Vasconcelos, On the arithmetic and homology of algebras of linear type, Trans. Amer. Math. Soc. 283 (1984), 661-683.

8. J. Herzog, A. Simis and W. Vasconcelos, Ideals with sliding depth, Nagoya Math. J. 99 (1985), 159-172.

9. T. Hà and N. Trung, Asymptotic behavior of arithmetically Cohen-Macaulay blow-ups, Trans. Amer. Math. Soc. 357 (2005), 3655-3672.

10. C. Huneke, Strongly Cohen-Macaulay schemes and residual intersections, Trans. Amer. Math. Soc. 277 (1983), 739-763.

11. M. Johnson and B. Ulrich, Artin-Nagata properties and Cohen-Macaulay associated graded rings, Compos. Math. 103 (1996), 7-29.

12. K-N Lin, Rees algebras of diagonal ideals, J. Comm. Alg. 5 (2013), 359-398.

13. A. Simis and B. Ulrich, On the ideal of an embedded join, J. Algebra 226 (2000), 1-14.

14. B. Sturmfels and S. Sullivant, Combinatorial secant varieties, Pure Appl. Math. 2 (2006), 867-891.

The Pennsylvania State University, Greater Allegheny, Academic Affairs, 4000 University Dr., McKeesport, PA 15132

Email address: kul20@psu.edu 